SCIENTIFIC REPORT

\title{
Multifocal VEP in children: its maturation and clinical application
}

\author{
C Balachandran, A I Klistorner, F Billson
}

Br J Ophthalmol 2004;88:226-232. doi: 10.1136/bjo.2003.018390

\begin{abstract}
Aim: To study the maturation of multifocal visual evoked potentials (multifocal VEP) in normal children between the ages of 5 and 16 years and to apply the results clinically in selected cases to the diagnosis of optic pathway diseases. Method: 70 normal children were recruited from the community and multifocal VEP (Accumap ObjectiVision, Sydney, Australia) was recorded. The waveform of the evoked responses, the latency and amplitude were analysed. Using these data, an age matched comparison was made with three children with advanced optic nerve disease; two had optic nerve glioma and one had congenital glaucoma. Results: The full field amplitude did not correlate with age and varied greatly within each age group (coefficient of variability $28 \%$ ). When scaled with respect to the background electroencephalogram the intra-age group variability decreased to $15 \%$ and a sigmoid relation was found between amplitude and age. The scaled amplitude remained largely unchanged till 11 years, between 11 and 13 years there was a rapid increase $(40 \%)$, and remained stable thereafter. This relation was seen at all eccentricities tested. The latency decreased gradually with age and plateaved at 13 years. In the three children with vision abnormalities this test was able to detect scotomas consistent with their condition.

Conclusion: Multifocal VEP perimetry shows an age related maturation in the visual pathway, characterised by distinctive timeframe of development for amplitude and latency. It can be performed by children as young as 5 years of age and holds promise as a diagnostic test capable of documenting children's visual fields objectively, even before they are able to perform subjective field tests.
\end{abstract}

M ultifocal visual evoked potential (VEP), a recent advancement in electrophysiology, has made it possible to document the visual field of an individual as a collection of evoked cortical responses. ${ }^{12}$ Multiple domains/loci of the visual field are simultaneously stimulated using a cortically scaled pseudorandomly reversing pattern stimulus. Visual evoked potentials corresponding to each of the loci of the visual field tested can be recorded within a short period of time to generate a perimetry of VEP. This technique has been successfully applied in adults to the detection of glaucoma and other diseases affecting the visual pathway..$^{2-5}$

Its objective nature, need for minimum cooperation from the subject and short recording time make it an ideal technique for investigating visual fields in children. To the best of our knowledge the application of this technique has not been extended to this age group. Furthermore, there is unresolved controversy about the maturation of VEP in school aged children. While a number of studies using conventional VEP techniques suggest that latency shows an age related decline in children older than 4 years, ${ }^{6-8}$ others show no change. ${ }^{10}$ Amplitude was also found to decrease with age in some studies, ${ }^{6810}$ while others were inconclusive. ${ }^{11}{ }^{12}$ Madrid and Crognale ${ }^{13}$ investigated the maturation of the $\mathrm{M}$ and $\mathrm{P}$ pathways in children using different stimulus paradigms. They found that the achromatic luminance stimulus thought to be mediated by the M pathway, achieved adult-like waveform in infancy, with a slight increase in amplitude until 10 years, which subsequently declined. The chromatic pattern onset VEP thought to be mediated by the $\mathrm{P}$ pathway, showed a more delayed maturation, undergoing a transition in the waveform between 6 and 14 years, ${ }^{13}{ }^{14}$ changing from a positive-negative waveform (age 2-6) to a triphasic wave (age 6-14) to adult-like negative-positive waveform thereafter. ${ }^{13}$ Others ${ }^{15}$ have also noted a significant increase in steady state VEP threshold at about 11 years of age when using high contrast high spatial frequency gratings ( $\mathrm{P}$ mediated) with no change in $\mathrm{M}$ pathway responses. Behavioural studies also support the continued maturation of luminance contrast, ${ }^{16}{ }^{17}$ colour contrast, ${ }^{18}$ visual acuity, ${ }^{19} 20$ and stereopsis ${ }^{21}$ in school aged children.

Therefore, the aims of the study were to investigate maturation of amplitude and latency of multifocal VEP, and possible application of this technique towards the detection of visual field defects in children.

\section{METHODS}

\section{Subjects}

Seventy normal children between the ages of 5 and 16 (40 females, 30 males, mean and median age 10 years), without a history of ocular or systemic disease were recruited from the community. The study protocol was approved by our regional ethics committee and adhered to the tenets of the Declaration of Helsinki. Informed consent was obtained from the parents of all subjects. Entry criteria were $6 / 6$ corrected visual acuity in both eyes and a normal ophthalmic examination. Both eyes were tested but only the left eye was analysed.

\section{Stimulus}

In brief, 58 closely packed segments in a cortically scaled dartboard of $4 \times 4$ checks (per segment) configuration were generated on a computer screen (22 Hitachi high resolution display, refresh rate $75 \mathrm{~Hz}$ ), to stimulate to a radial eccentricity of $32^{\circ}$ (fig 1 , for more details see Goldberg et $a l^{4}$ and Ossenblok et $a l^{22}$ ). Each segment contained a checkerboard pattern with the size of individual checks being proportional to the size of the segment. Luminance of the white check was $146 \mathrm{~cd} / \mathrm{m}^{2}$ and luminance of the black check $1.1 \mathrm{~cd} / \mathrm{m}^{2}$, producing Michelson contrast of $99 \%$. Background luminance of the screen was maintained at a mean level of $73.5 \mathrm{~cd} / \mathrm{m}^{2}$. A dim room light was always on.

The reversal of the 58 segments was controlled by 58 binary pseudorandom sequences (with 1024 elements/reversals) derived from a family of sequences called Kasami sequences. ${ }^{23}$ At the end of the sequences, which lasted 


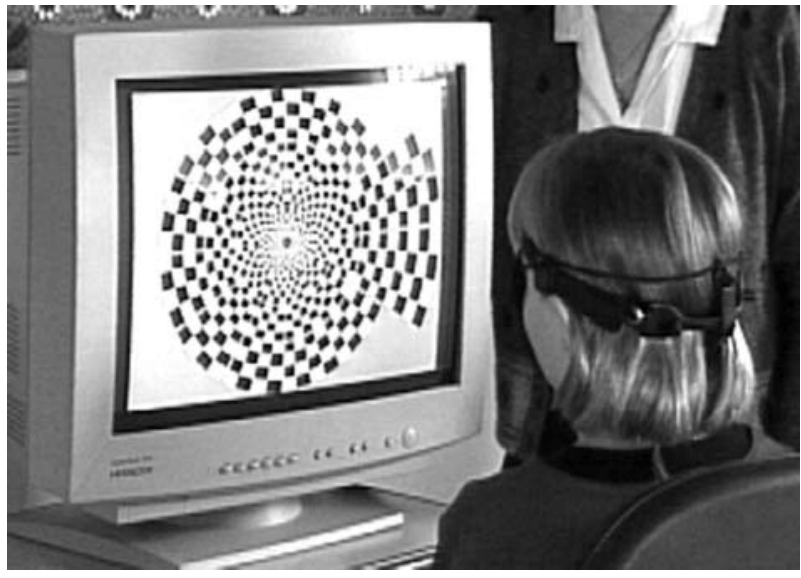

Figure 1 Photograph of a 10 year old child performing the test.

13 seconds in total (also called a run), the sequences were correlated with the electrical responses recorded and the VEP signal was extracted. Subsequent runs then used different sequences for the same segment.

\section{Recording}

The child was seated $30 \mathrm{~cm}$ from the stimulus monitor. The central area of the stimulus (radius $1^{\circ}$ ) was not used for stimulation; it contained a fixation target, which changed randomly every 3 seconds between the digits 3, 5, 6, 8, and 9 . Children were instructed to press a button when they saw a nominated number. This allowed fixation and attention to be monitored during the test. If there was more than one fixation error, the run was re-recorded after a period of rest.

Data were recorded using bipolar occipital inion straddle electrodes using Accumap system (Objectivision Pty Ltd, Sydney, Australia). It consists of two electrodes positioned $4 \mathrm{~cm}$ from the inion on either side of midline and two placed $2.5 \mathrm{~cm}$ above and $4.5 \mathrm{~cm}$ below the inion in the midline. Electrical signals were recorded along four channels; each channel being the difference in electrical signal between two electrodes. The vertical and horizontal channel recordings were derived from the vertical and horizontal electrodes respectively. The left and right oblique channels were recorded as the difference between the lower electrode and the left and right horizontal electrodes respectively. A ground electrode was placed on the right or left ear lobe.

The eye was stimulated for a total of 10 minutes (40 runs, each 13 seconds long) and the data were recorded using Grass 4 channel amplifier Model 15 Neurodata (Astro-Med Inc, Rhode Island, USA). The signal was amplified 100000 times and band pass filtered between 1 and $30 \mathrm{~Hz}$.

\section{Analysis}

VEP traces were analysed using Opera software (Objectivision Pty Ltd, Sydney, Australia). The study of the VEP waveform and latency analysis, was limited to the vertical channel, as the polarity of the responses varied between the four channels. Unfortunately, since vertical channel recording alone does not reliably detect responses along the horizontal and upper vertical meridians owing to the orientation of the visual cortex relative to the dipole of the vertical channel, a signal was not always present in all 58 domains. Therefore, latency could not be analysed for individual segments or concentric rings and was limited to the hemifield.

The analysis of amplitude took advantage of all four channels. Largest peak to trough amplitudes for each wave within the interval of $60-180 \mathrm{~ms}$ were determined and

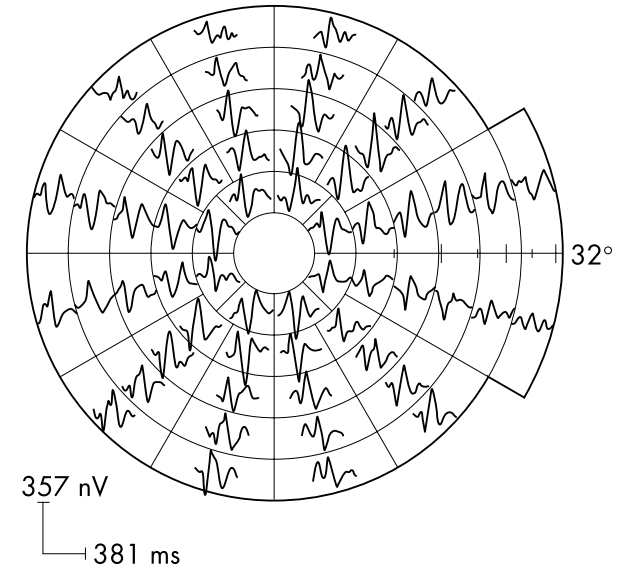

Figure 2 Left eye combined topographic map recorded from a 6 year old child using four channel recording. Upward deflections on the traces represent positive peaks.

compared among channels for every stimulated segment of the visual field. The wave of maximal amplitude from each point in the field, from the four channels (vertical, horizontal, right, and left oblique) was automatically selected and the software created a combined topographic map (fig 2). ${ }^{24}$ The combined topographic map was then used for further amplitude analysis.

The background electroencephalographic (EEG) activity was estimated using a Fourier power spectrum (fast Fourier transform) of the raw data (fig 3). The Fourier power spectrum quantifies the amount of brain activity (in arbitrary units) at different frequencies that together constitute the EEG. The area under this curve is a measure of the total EEG generated (in arbitrary units). This was measured by fitting the curve with a mathematical polynomial function described in detail, elsewhere. ${ }^{25}$

\section{RESULTS}

\section{$M-V E P$ waveform and latency}

The waveforms mirrored each other in the superior and inferior fields (fig 4). In both regions there was a gradual age related narrowing of the waveforms. The latency of the dominant peak negative in superior field and positive in inferior field showed a decrease with age reaching a plateau at 13 years of age (fig 5). The second positive peak in the superior field at $235 \mathrm{~ms}$ showed a decrease in amplitude with age, although a similar change in the negative peak was not found in the inferior field (fig 4).

\section{Amplitude}

There was a large variation in full field amplitude between children, with no significant correlation with age (linear correlation coefficient, $-0.21,95 \%$ CI -0.45 to 0.03 , fig $6 \mathrm{~A}$ ). The children were divided into 3 year age groups and the coefficient of variability within a group was found to be $28 \%$ (range $25 \%-30 \%$ ). Unlike amplitude and age, there was a

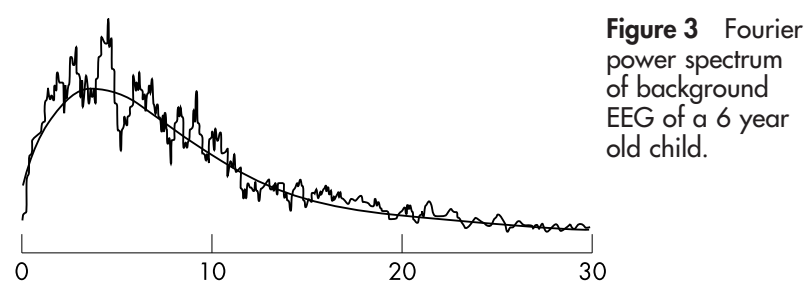



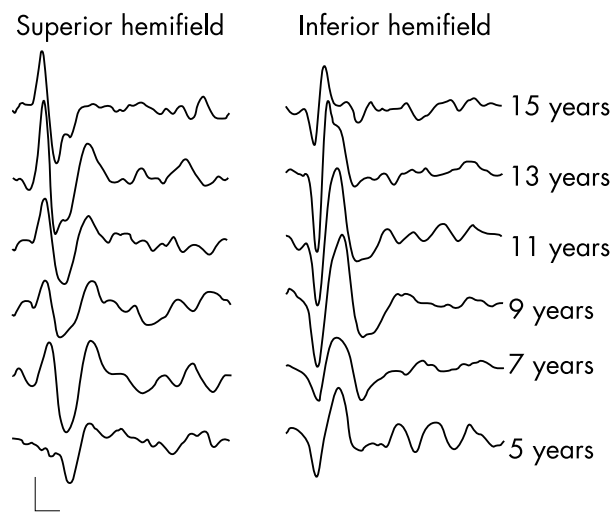

Figure 4 Multifocal VEP traces of the superior and inferior hemifields from the vertical channel. Traces shown are example of VEP with best signal to noise ratio. Vertical and horizontal scales represent $100 \mathrm{nV}$ and $100 \mathrm{~ms}$ respectively. Upward deflections on the traces represent positive peaks.

significant correlation between full field amplitude and EEG Fourier power (fig 6B) linear correlation coefficient $0.61,95 \%$ CI 0.42 to 0.8 ). When scaled with respect to the EEG, the intra-age group variability in amplitude decreased by more than $45 \%$ (fig 6C) coefficient of variability $15 \%$, range $13 \%-$ $18 \%$ ) and a very clear sigmoid relation was found between amplitude and age. There was a $40 \%$ increase in amplitude between 11 and 13 years of age.

The maturation of amplitude was examined at different eccentricities of the visual field by analysing each ring of the stimulus separately. In each ring the normalised amplitude showed a sigmoid increase with age, similar to the average full field amplitude (fig 6D). This may indicate that maturation occurs at the same rate at different eccentricities.

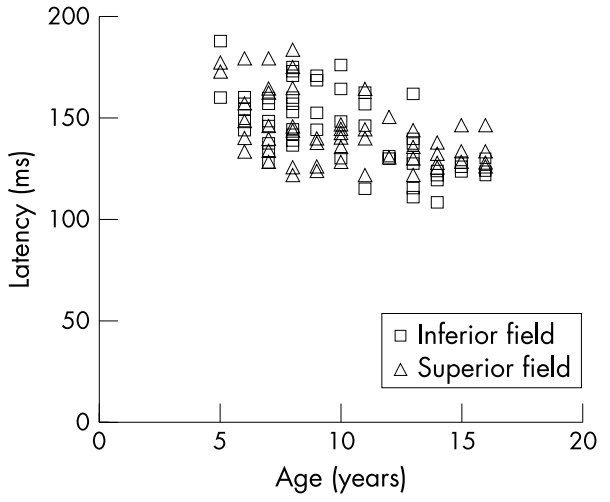

Figure 5 Hemifield latency with age.

\section{Clinical case examples}

A 6 year old child with a large optic nerve glioma (neurofibromatosis negative) involving the left optic nerve and chiasm was tested. Her visual acuity was 6/6 in the right eye and hand movement in the left eye. She was unable to perform subjective perimetry but was able to perform m-VEP (fig 7A). Her m-VEP amplitudes were compared with age matched normal children and an amplitude deviation or probability plot was produced. A reproducible absolute defect involving the entire left eye and the superior temporal field of the right eye was noted (fig 7B). There was no change in her visual field over a l year period, which was consistent with the radiological findings.

An 11 year old child with post-resection of tumour involving the right optic nerve and chiasm (visual acuity no light perception RE, 6/6 LE) was also tested. A temporal hemianopia respecting the vertical meridian was seen in the LE (fig 8).
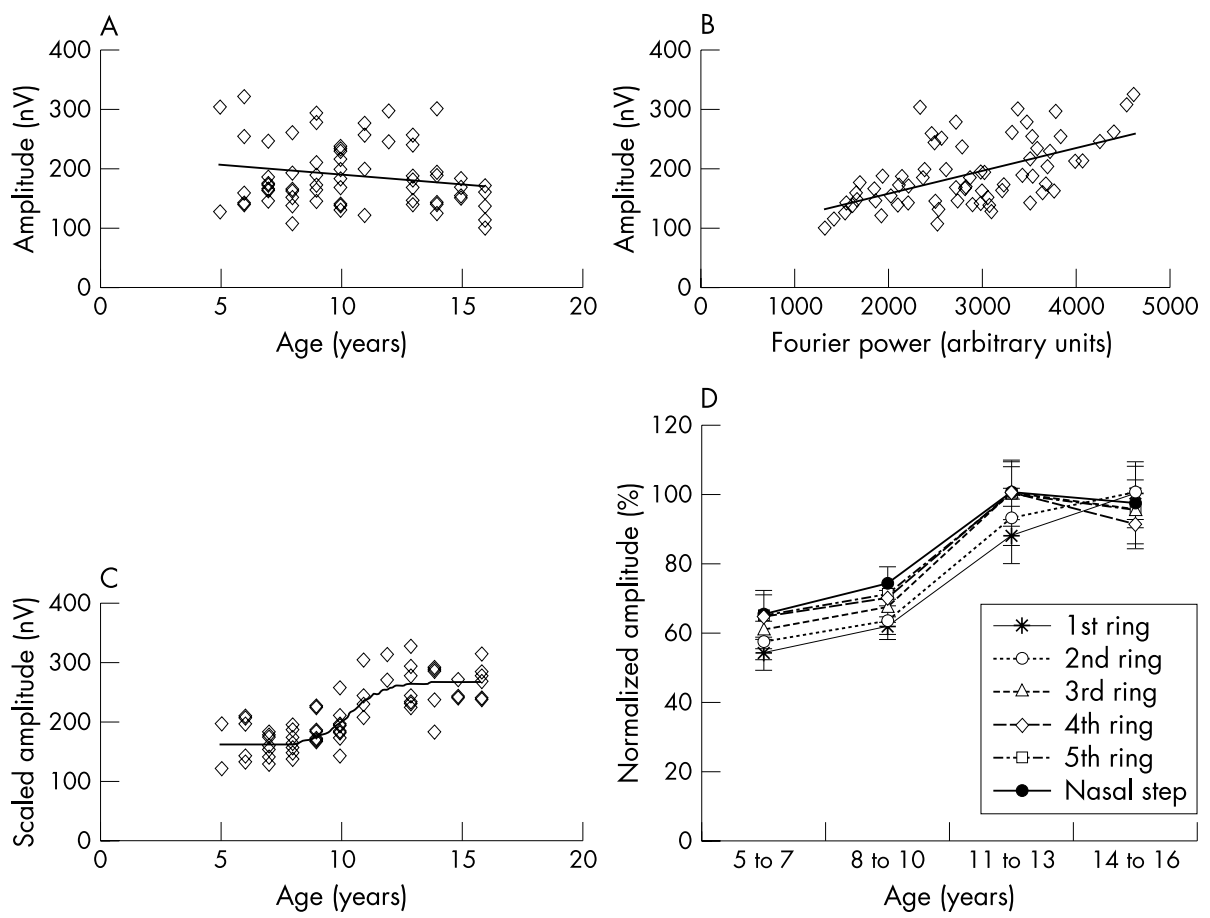

Figure 6 (A) Scatter plot of full field amplitude and age. (B) Scatter plot of linear correlation between EEG Fourier power and age. (C) Scatter plot of age and full field amplitude scaled with respect to background EEG Fourier power. Fitted with a sigmoid curve. (D) Plot of normalised amplitude at different eccentricities with age. The age groups of 5-7, 8-10, 11-13, 14-16 years, contained 15, 26, 14, 15 children respectively. 


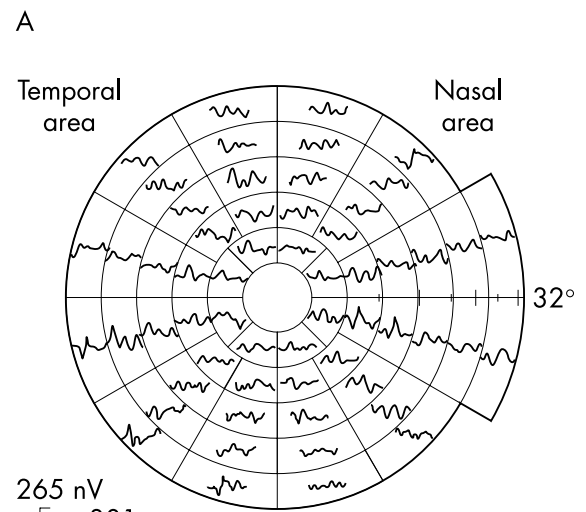

$[381 \mathrm{~ms}$
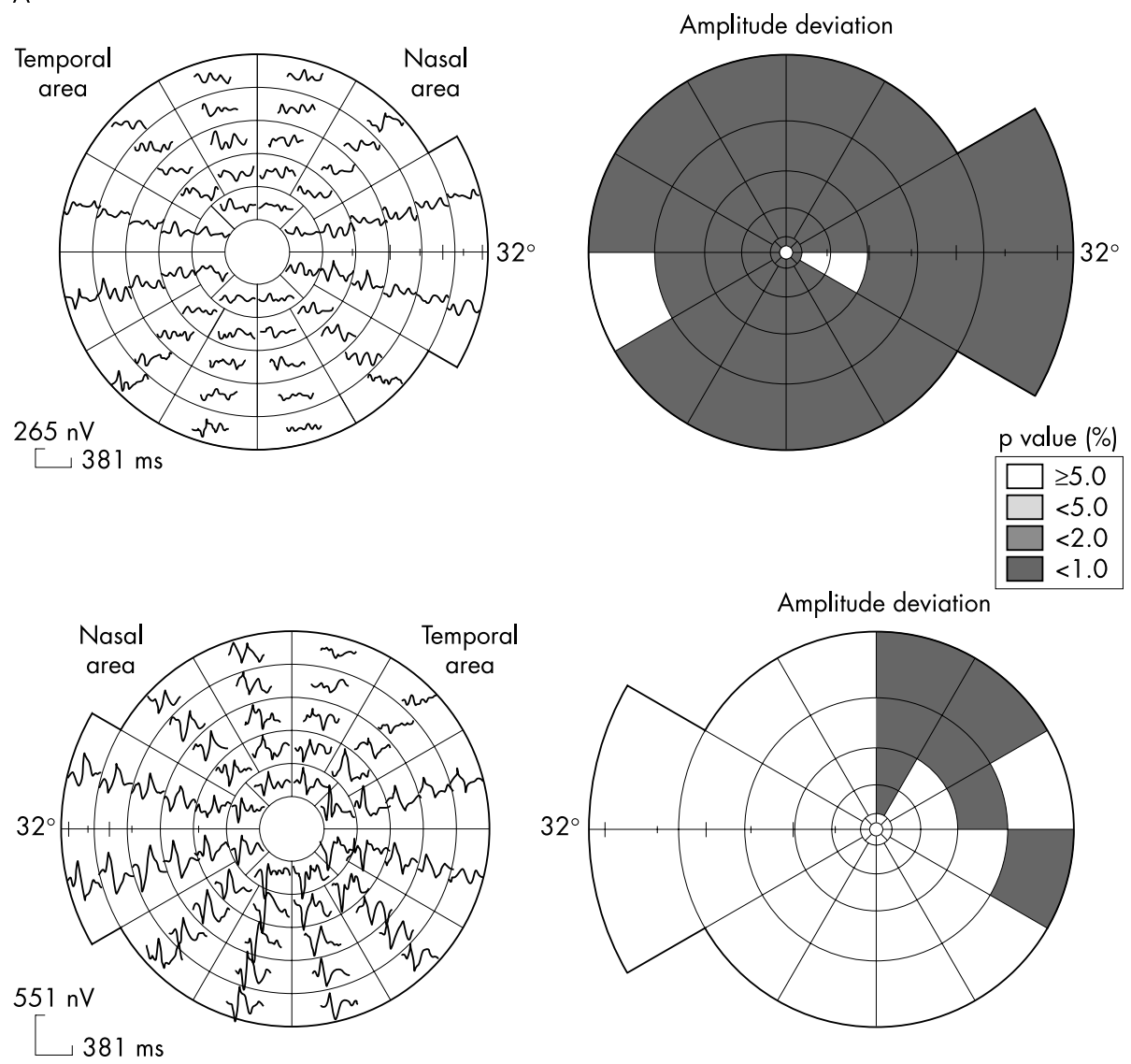

B
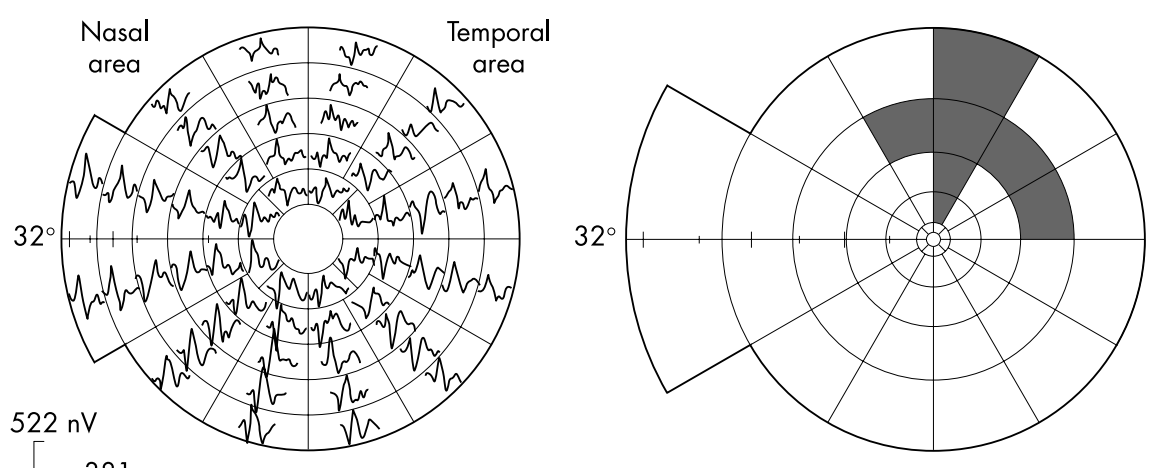

Figure 7 Multifocal VEP recording of a 6 year old child with optic nerve glioma involving the left optic nerve and chiasm, who was unable to perform subjective perimetry. (A) The right and left eye multifocal VEP traces and the age matched comparisons are shown. (B) The scotoma was reproduced on repeat testing.

Lastly, a child (age 11 years) suffering from glaucoma secondary to Sturge-Weber syndrome was examined. The amplitude deviation plot did not detect any defect because of the large variability in the normal sample in this age group. Thus, the inter-eye difference in amplitude divided by the sum of the amplitudes was calculated for each location. This asymmetry coefficient was compared with that derived from the age matched normal sample to generate the inter-eye asymmetry plot (fig 9B), which shows a scotoma consistent with the defect noted on subjective perimetry (fig 9A).

\section{DISCUSSION}

The current study suggests that the multifocal VEP, measured with the Accumap, shows a period of marked change between 10 and 13 years. This finding lends further support to the behavioural and electrophysiological evidence that the visual system continues to mature beyond the age of 5 years. Furthermore, the present study introduces the possibility of detecting visual field abnormalities in children who are unable to perform subjective perimetry, using the m-VEP technique.

In the mature visual system, Klistorner and Graham ${ }^{25}$ found that the large intersubject variability in multifocal VEP amplitude $(40 \%)$ was markedly reduced $(24 \%)$ when EEG scaling was introduced. Furthermore, in adults the scaled amplitude was found to be independent of age. This scaling was postulated to be compensating for differential conductance across the meninges, skull, and skin. 

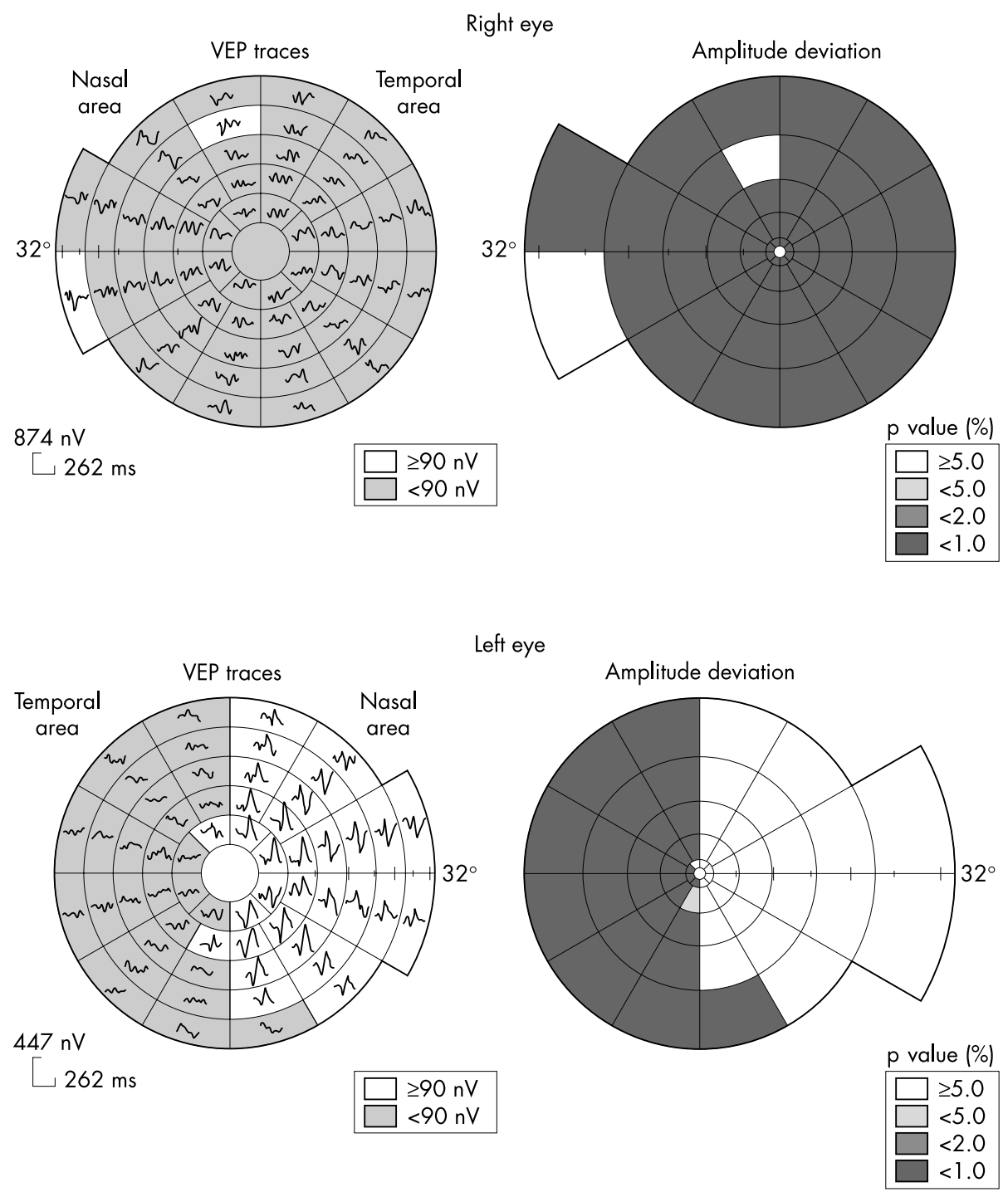

Figure 8 Multifocal VEP recording of an 11 year old child with post-resection of an optic nerve glioma involving her right optic nerve and chiasm. Her visual acuity was $6 / 12$ in the left eye and she was unable to perceive light with the right eye.

In children, although EEG scaling reduced amplitude variability to that seen in adults, a marked increase in scaled amplitude was found between 11 and 13 years at all eccentricities. This dramatic increase is reflected in a sigmoid relation (fig 6). The multifocal VEP latency does not show similar periods of marked change; however, it too gradually improves to plateau at 13 years, coincident with the maturation of amplitude. These findings suggest an increasing complexity of physiological response during maturation, extending at least into early adolescence.

The source of this rise in amplitude and decrease in latency can only be hypothesised. Using dipole source localisation technique, maturation has been postulated to occur in both striate and extrastriate cortices. ${ }^{22}{ }^{26}$ It is possible that there is greater recruitment of neurons in the striate cortex producing the increase in scaled amplitude without similar change in latency, although the synaptic density is known to reach adult levels by 11 years of age. ${ }^{27}$ Furthermore, although this age group is not characterised by large changes in head circumference it is possible that there is a change in intracranial striate cortex morphology, which is providing better exposure to the dipoles of the recording electrode.
The current stimulus paradigm does not selectively stimulate the $\mathrm{M}$ or $\mathrm{P}$ pathway. Although, it is likely that the high contrast, high spatial frequency attributes favour the $\mathrm{P}$ pathway, the high temporal frequency would probably favour the $\mathrm{M}$ pathway. There is a greater role for the $\mathrm{P}$ pathway in central vision with the $\mathrm{M}$ pathway contribution increasing towards the periphery. Our results did not reveal any differential maturation with eccentricity, suggesting that both pathways mature at the same rate. However, since our stimulus is not optimised to differentiate between the two pathways, further studies need to be done to delineate the $M$ and $\mathrm{P}$ pathways using the multifocal VEP technique.

The inter-individual variation within an age group during maturation could reflect different rates of maturation between individuals. If this is the case then the value of objective visual field testing will have its greater strength in the longitudinal follow up of individual cases, particularly where there is suspect pathology and there is a need to determine whether it is progressive or static. Three illustrative cases with advanced visual field defects are referred to. Whilst a larger database is needed to accurately detect visual field loss, this study suggests that this form of testing is feasible in children. 
A

$$
\begin{array}{rllll|llll} 
& <0 & 10 & 12 & 16 & 18 & 17 & \\
& 9 & 21 & 28 & 20 & 19 & 21 & 16 & 20 \\
30+\frac{10}{0} & 10 & 26 & 26 & 28 & 31 & 29 & 21 & 29 \\
\hline 23 & 28 & 31 & 31 & 31 & 32 & 32 & <0 & 31 \\
27 & 28 & 30 & 30 & 32 & 32 & 32 & 30 \\
& 26 & 30 & 29 & 30 & 30 & 28 & \\
& & 31 & 29 & 28 & 30 & &
\end{array}
$$
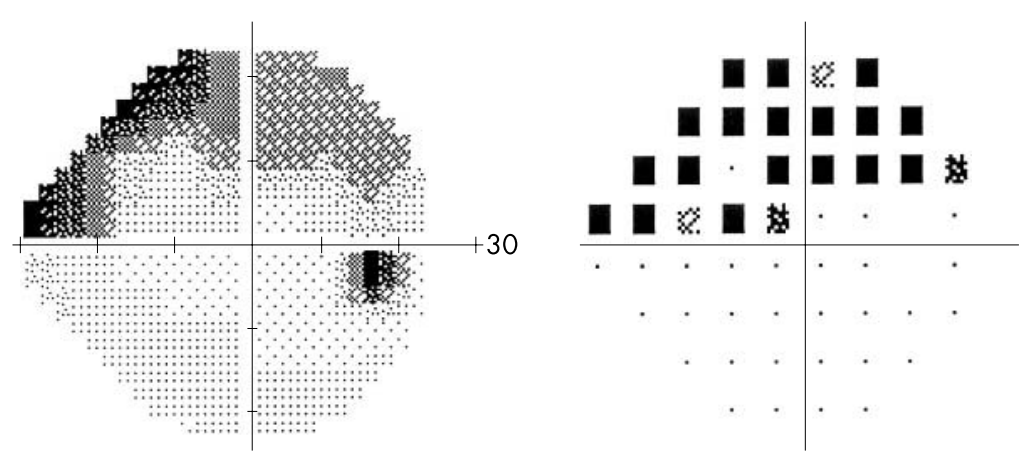

B
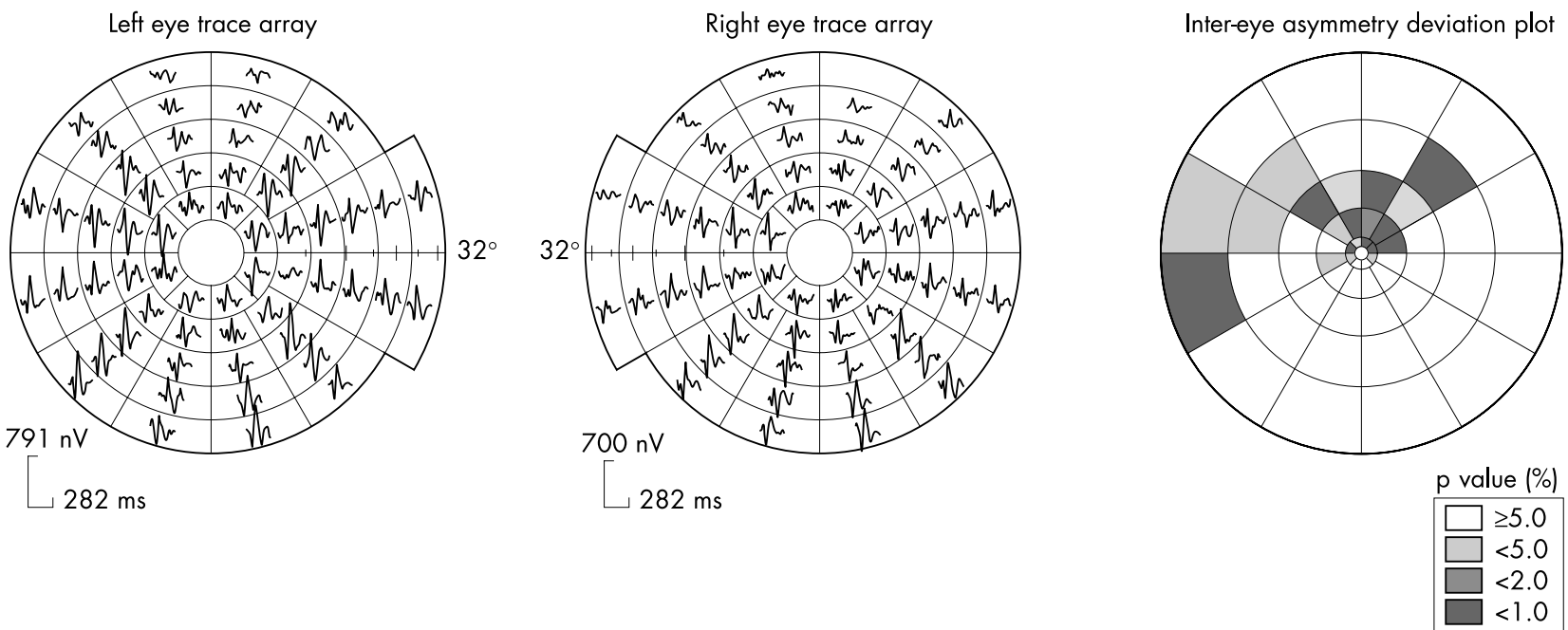

Figure 9 (A) Subjective field results of an 11 year old child with glaucoma secondary to Sturge-Weber syndrome. (B) Multifocal VEP recordings from the same child, showing a superior field defect on the inter-eye asymmetry deviation plot.

In summary, the multifocal VEP is a test that can be performed by children as young as 5 years of age. It shows age related maturation over the entire visual field and further research is needed to delineate the different components undergoing maturation. Lastly, this test holds promise in being able to diagnose and monitor visual field defects in young children.

\section{Authors' affiliations}

C Balachandran, A I Klistorner, F Billson, Save Sight Institute,

Department of Ophthalmology, University of Sydney, Sydney, Australia

Correspondence to: $\operatorname{Dr} C$ Balachandran, Save Sight Institute, Sydney Eye Hospital, Macquarie St, PO Box 1614, Sydney 2001, Australia; Chandra@eye.usyd.edu.au

Accepted for publication 12 May 2003

\section{REFERENCES}

1 Baseler HA, Sutter EE, Klein SA, et al. The topography of visual evoked response properties across the visual field. Electroencephal Clin Neurophysiol 1994; $90: 65-81$

2 Klistorner Al, Graham SL, Grigg JR, et al. Multifocal topographic visual evoked potential: improving objective detection of local visual field defects. Invest Ophthalmol Vis Sci 1998;39:937-950.

3 Hood DC, Odel JG, Zhang X. Tracking the recovery of local optic nerve function after optic neuritis: a multifocal VEP study. Invest Ophthalmol Vis Sci 2000:41:4032-8.

4 Goldberg I, Graham SL, Klistorner A. Multifocal objective perimetry in the detection of glaucomatous field loss. Am J Ophthalmol 2002;133:29-39.

5 Klistorner Al, Graham SL, Grigg JR, et al. Multifocal topographic visual evoked potential: improving objective detection of local visual field defects. Invest Ophthalmol Vis Sci 1998;39:937-50.
6 Allison T, Wood CC, Goff WR. Brain stem auditory, pattern-reversal visual, and short-latency somatosensory evoked potentials: latencies in relation to age, sex, and brain and body size. Electroencephalogr Clin Neurophysiol 1983:55:619-36.

7 Emmerson-Hanover R, Shearer DE, Creel DJ, et al. Pattern reversal evoked potentials gender differences and age-related changes in amplitude and latency Electroencephalogr Clin Neurophysiol 1994;92:93-101.

8 Breceli J, Strucl M, Zidar I, et al. Pattern ERG and VEP maturation in schoolchildren. Clin Neurophysiol 2002;113:1764-70.

9 Shaw NA. Changes in the cortical components of the visual evoked potential with age in man. Austr J Exp Biol Med Sci 1984;62(Pt 6):771-8.

10 Snyder E, Dustman R, Shearer D. Pattern reversal evoked potential amplitudes: lifespan changes. Electroencephalogr Clin Neurophysiol 1981;52:429-34

11 Fenwick P, Brown D, Hennesey J. The visual evoked response to pattern reversal in 'normal' 6-11-year-old children. Electroencephalogr Clin Neurophysiol 1981;51:49-62.

12 Aso K, Watanabe K, Negoro T, et al. Developmental changes of pattern reversal visual evoked potentials. Brain Dev 1988;10:154-9.

13 Madrid MA, Crognale MA. Longterm maturation of visual system. Vis Neurosci 2000;17:831-7.

14 Crognale MA, Kelly JP, Weiss AH, et al. Development of the spatio-chromatic visual evoked potential (VEP): a longitudinal study. Vis Res 1998;38:3283-92.

15 Gordon GE, McCulloch DL. A VEP investigation of parallel visual pathway development in primary school age children. Doc Ophthalmol 1999;99:1-10.

16 Bradley A, Freeman RD. Contrast sensitivity in children. Vis Res 1982;22:953-9.

17 Hainline L, Abramov I. Development of spatial contrast sensitivity from infancy to adulthood: psychophysical data. Optometry Vis Sci 1997;74:785-9.

18 Knoblauch K, Vital-Durand F, Barbur JL. Variation of chromatic sensitivity across the life span. Vis Res 2001;41:23-36.

19 Schmid M, Largo RH. Visual acuity and stereopsis between the ages of 5 and 10 years. A cross-sectional study. Eur J Pediatr 1986;145:475-9.

20 Hohmann A, Haase W. Development of visual line acuity in humans. Ophthalmic Res 1982;14:107-12. 
21 Romano PE, Romano JA, Puklin JE. Stereoacuity development in children with normal binocular single vision. Am J Ophthalmol 1975;79:966-71.

22 Ossenblok P, Reits D, Spekreijse H. Analysis of striate activity underlying the pattern onset VEP of children. Vis Res 1992;32:1829-35.

23 Sarwate, Pursley. Crosscorrelation properties of pseudorandom and related sequences. Proc IEEE 1980;68:593-619.

24 Klistorner A, Graham SL. Objective perimetry in glaucoma. Ophthalmology 2000;107:2283-99.
25 Klistorner A, Graham SL. Electroen ephalogram-based scaling of multifocal visual evoked potentials: effect on intersubject amplitude variability. Invest Ophthalmol Vis Sci 2001;42:2145-52.

26 Ossenblok P, De Munck JC, Wieringa HJ, et al. Hemispheric asymmetry in the maturation of the extrastriate checkerboard onset evoked potential. Vis Res 1994;34:581-90.

27 Garey L. Structural development of the visual system of man. Human Neurobiol 1984;3:75-80.

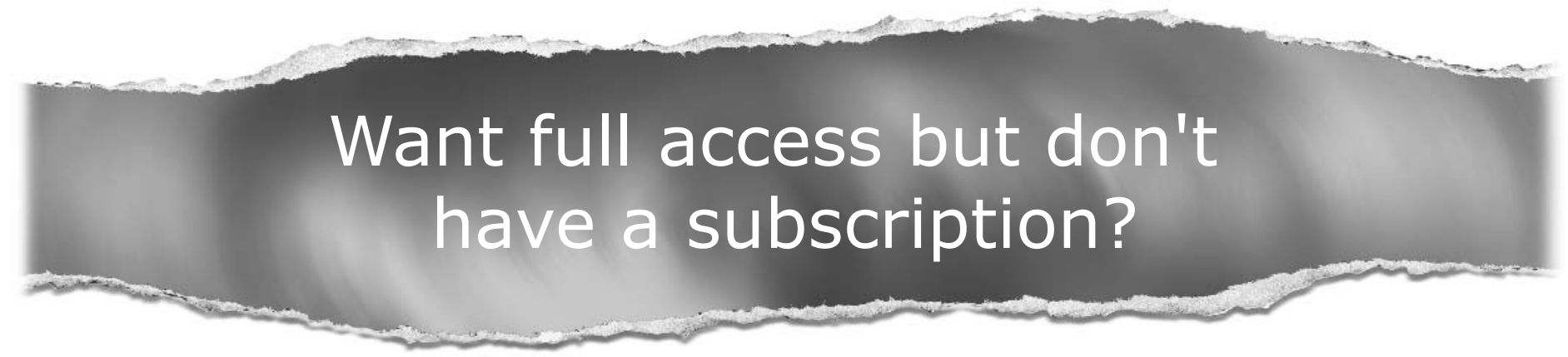

\section{Pay per access}

For just US $\$ 25$ you can have instant access to the whole website for 30 days. During this time you will be able to access the full text for all issues (including supplements) available. You will also be able to download and print any relevant pdf files for personal use, and take advantage of all the special features British Journal of Ophthalmology online has to offer.

www.bjophthalmol.com 